Louisiana State University

LSU Digital Commons

Faculty Publications

Department of Chemistry

8-8-2017

\title{
Coarse-Grained Models of Aqueous and Pure Liquid Alkanes
}

\author{
Gaurav Gyawali \\ University of New Orleans \\ Samuel Sternfield \\ University of New Orleans \\ Revati Kumar \\ Louisiana State University \\ Steven W. Rick \\ University of New Orleans
}

Follow this and additional works at: https://digitalcommons.Isu.edu/chemistry_pubs

\section{Recommended Citation}

Gyawali, G., Sternfield, S., Kumar, R., \& Rick, S. (2017). Coarse-Grained Models of Aqueous and Pure Liquid Alkanes. Journal of Chemical Theory and Computation, 13 (8), 3846-3853. https://doi.org/10.1021/

acs.jctc. $7 b 00389$

This Article is brought to you for free and open access by the Department of Chemistry at LSU Digital Commons. It has been accepted for inclusion in Faculty Publications by an authorized administrator of LSU Digital Commons.

For more information, please contact ir@lsu.edu. 


\title{
Coarse grained models of aqueous and pure liquid alkanes
}

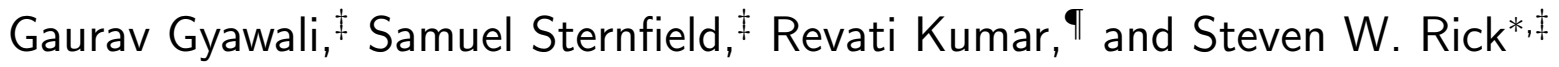 \\ $\ddagger$ Department of Chemistry, University of New Orleans, New Orleans, LA, 70148 \\ IDepartment of Chemistry, Louisiana State University, Baton Rouge, LA, 70808 \\ E-mail: srick@uno.edu
}

\begin{abstract}
A model for linear alkanes is presented in which interaction sites are only on the carbon atoms and the range of the potential is reduced using the StillingerWeber potential. The model is optimized for aqueous and liquid alkane properties and can match thermodynamic and structural properties, including solvation free energies, liquid densities, and liquid/vapor and liquid/water surface tensions for alkanes over a range of lengths. The results for long alkanes indicates that such models can be useful as accurate, yet efficient, coarse-grained potentials for macromolecules in water and other environments.
\end{abstract}

\section{Introduction}

The assembly of hydrophobic macromolecules in water involves a range of length scales, from the Angstrom scale of a water molecule to the tens of nanometer scale 
of the solute, a range of interactions from strong water-water interactions to the weaker interactions between non-polar groups, and a range of time scales. ${ }^{1}$ These effects combine to present a challenge for molecular simulations. Coarse-grainied (CG) models, in which atomic interaction sites are combined into larger groups, can be used to access larger length and time scales. Changing the range of the potential, so that only interactions between a relatively small number of particles remain, represents another method to gain efficiency. The combination of $\mathrm{CG}$ and reduced range $(\mathrm{RR})$ is used in the Stillinger-Weber potential, ${ }^{2}$ originally developed to model silicon and extended to models for water, ${ }^{3-5}$ ions in water, ${ }^{6}$ and methane in water ${ }^{7}$ by Molinero and co-workers. The method is able to treat systems with electrostatic interactions using only short-ranged potentials, resulting in models that are two to three orders of magnitude more efficient than all-atom models, with only elimination of hydrogen sites. ${ }^{3}$

United atom (UA) models for alkanes, which eliminate interactions on non-polar hydrogens, include those of Ryckaert and Bellemans, ${ }^{8}$ Weber $^{9}$ and the OPLS-UA model of Jorgensen, Madura, and Swenson. ${ }^{10}$ The development of UA models for alkanes ${ }^{11-16}$ and proteins ${ }^{17}$ continues. These models use conventional Lennard-Jones and Coulombic interactions, on polar atoms, and are meant to be used with all-atom water models. A number of larger-scale CG models for alkanes, which map about four heavy atoms onto one CG site (4:1 mapping) have been developed. ${ }^{18-23}$ These models typically use Lennard-Jones and electrostatic interactions between the CG particles, ${ }^{24}$ although other potentials forms like the Morse potential ${ }^{23}$ or a numerical $\operatorname{grid}^{21}$ are also used.

An interesting comparison of the gains from $\mathrm{CG}$ versus $\mathrm{RR}$ can be made using liquid water as an example. If the number of particles that a given molecule interacts with is $\mathrm{N}_{n b r}$, then the number of interacting particles is $(1 / 2) \mathrm{N} \mathrm{N}_{n b r}$ where $\mathrm{N}$ is the 
number of particles and the factor of $1 / 2$ is to avoid over-counting. Many models use a $12 \AA$ cut-off, and at the density of water at room temperature and pressure, this gives $\mathrm{N}_{n b r}$ about 240. A 4:1 CG model would then have $\mathrm{N}_{n b r}$ equals 240 divided by 4, or 60. The Molinero and Moore Stillinger-Weber water model $(\mathrm{mW})^{3}$ explicitly goes to zero at $4.31 \AA$, giving $\mathrm{N}_{n b r}$ of about 10 . The $\mathrm{mW}$ water model then has $5 \mathrm{~N}$ interactions and a 4:1 CG model would have $(1 / 2)(\mathrm{N} / 4) 60$ or $7.5 \mathrm{~N}$, since the 4:1 CG model would have 4 times fewer particles in the same volume. For comparison, a 3-site all-atom model would have $1080 \mathrm{~N}\left(3^{2} \times 240 \times \mathrm{N} / 2\right)$ interactions. So the $\mathrm{mW}$ RR and the 4:1 CG models have similar efficiencies and in this case the RR model is a little better. (This assumes the potentials are equally expensive to calculate, ignores the cost of Ewald sums and other long-ranged corrections that may be used, and does not consider the different time steps used.) This suggests the question, is it better to lose atomic resolution but retain long-ranged interactions, or keep the atomic scale and lose long-ranged interactions? The first approach is coarse graining on the local level and the second could be thought of as coarse graining on the distant level. ${ }^{25}$ These two approaches are coupled, since CG potentials get longer ranged as the level of coarsening increases ${ }^{26,27}$

The importance of long-ranged interactions has received considerable attention for some time. ${ }^{28-32}$ It is clear that for potentials constructed to include long-ranged electrostatic interactions, it is important to have a proper treatment of those interactions, particularly for non-isotropic systems. ${ }^{33,34}$ More recently, it appears that effective shorter-ranged potentials can be constructed with careful adjustment of the potential, through local molecular field (LMF) theory ${ }^{35,36}$ or force-matching. ${ }^{25}$ The mW model differs from those approaches not only by being much shorter-ranged, but also starting from a new potential form, rather than mapping backwards from long-ranged potentials. Much of the understanding of long- versus short-ranged inter- 
actions has focused on pair potentials. For example, it has been shown that single-site pair potentials cannot simultaneously reproduce the structural and thermodynamical properties of water. ${ }^{37,38}$ Given the highly anisotropic nature of water, the failure of one-site pair potentials is not that surprising. A critical part of the $\mathrm{mW}$ potential is the inclusion of three-body terms which establishes a tetrahedral local structure and the desired coordination number. Other accurate one-site water models have been developed through the inclusion of higher order electrostatic moments. ${ }^{39}$ Results from two-body CG models suggest that local structure is determined by local interactions while longer ranged interactions are necessary for thermodynamic properties like pressure. ${ }^{26,27,40}$ To overcome the two-body approximation, there are a variety of CG models that include many-body effects, ${ }^{41-43}$ in addition to the SW approach.

In this work, we aim to develop SW-type models for linear alkanes, as a step towards developing models for polymers and macromolecules. This work will focus on constructing models that are accurate for thermodynamic properties of liquid and aqueous alkanes.

\section{Methods}

The Stillinger-Weber potential is given by a sum of two body, $\phi_{2}$, and three body terms, $\phi_{3}$, as given by

$$
E=\sum_{i} \sum_{j>i} \phi_{2}\left(r_{i j}\right)+\sum_{i} \sum_{j \neq i} \sum_{k>j} \phi_{3}\left(r_{i j}, r_{i k}, \theta_{j i k}\right)
$$


with the two-body term given by

$$
\phi_{2}\left(r_{i j}\right)= \begin{cases}A \epsilon\left[B\left(\sigma / r_{i j}\right)^{p}-\left(\sigma / r_{i j}\right)^{q}\right] \exp \left(\sigma /\left(r_{i j}-a \sigma\right)\right) & \text { if } r_{i j}<a \sigma \\ 0 & \text { if } r_{i j}>a \sigma\end{cases}
$$

with p equals 4 and q equals 0 . The two body term is characterized by a well-depth, $\epsilon$, and a length scale, $\sigma$. The three body term is $\phi_{3}\left(r_{i j}, r_{i k}, \theta_{j i k}\right)=$

$$
\begin{cases}\lambda \epsilon\left[\cos \theta_{j i k}-\cos \theta_{0}\right]^{2} \exp \left(\gamma \sigma /\left(r_{i j}-a \sigma\right)\right) \exp \left(\gamma \sigma /\left(r_{i k}-a \sigma\right)\right) & \text { if } r_{i j} \text { and } r_{i k}<a \sigma \\ 0 & \text { if } r_{i j} \text { or } r_{i k}>a \sigma\end{cases}
$$

and acts between particle $i$ and its two neighbors $j$ and $k$, where $\theta_{j i k}$ is the angle between those three particles, $\theta_{0}$ is the target value of that angle, and $\lambda$ scales the strength of $\phi_{3}$. The three body term is necessary for systems with highly directional interactions, like hydrogen bonds. For the $\mathrm{mW}$ water model, $\theta$ equals $109.47^{\circ}$, which promotes a tetrahedral local structure. The parameters A,B, a, and $\gamma$ were optimized for silicon, ${ }^{2}$ and also shown to be optimal for water. ${ }^{44}$ As in previous studies,,${ }^{3,6,7}$ we do take these paramters as adjustable. $(\mathrm{A}=7.049556277, \mathrm{~B}=0.6022245583, \mathrm{a}=1.80$ and $\gamma=1.20$ ) In addition to being shorter ranged, $\phi_{2}$, is also less steeply repulsive than the Lennard-Jones potential (Figure 1), since it depends on $\mathrm{r}^{-4}$ rather than $\mathrm{r}^{-12}$.

Torsional energies used the Fourier form,

$$
E(\theta)=V_{0}+\frac{1}{2} V_{1}(1+\cos \phi)+\frac{1}{2} V_{2}(1-\cos 2 \phi)+\frac{1}{2} V_{3}(1+\cos 3 \phi)
$$


and the parameters are fit to the ab initio results (at the MP2/aug- cc-pVTZ level) for the torsional energy profile of hexane, as reported by Siu et al. ${ }^{45}$ In all-atom models, using torsional energies to fit these quantum results led to the correct percentage of gauche versus trans dihedral angles, ${ }^{45}$ which infrared spectroscopy experiments indicate should be about $33 \%{ }^{46}$ Our fit for the $\mathrm{CG}$ model is given by $\mathrm{V}_{0}=0, \mathrm{~V}_{1}=1.97$ $\mathrm{kcal} / \mathrm{mol}, \mathrm{V}_{2}=-1.16 \mathrm{kcal} / \mathrm{mol}$, and $\mathrm{V}_{3}=3.34 \mathrm{kcal} / \mathrm{mol}$. For the bond stretch, $E=$ $k_{b}\left(r-r_{0}\right)^{2}$, and bond angle terms, $E=k_{\theta}\left(\theta-\theta_{0}\right)^{2}$, we use the OPLS-UA values $\left(k_{b}=268 \mathrm{kcal} / \mathrm{mol} \AA^{-2}, r_{0}=1.529 \AA, k_{\theta}=58.35 \mathrm{kcal} / \mathrm{mol}\right.$, and $\left.\theta_{0}=112.7\right)$.

Parameters for the SW potential, like other CG potentials, can be developed by "bottom-up" approaches, in which the potential is set to match forces ${ }^{4,5}$ or to minimize the relative entropy ${ }^{47}$ of an all-atom model. Alternatively "top-down" approaches, in which the potential is set to match experimental properties can be used. $^{3,7}$ A combination of molecular level data from all-atom simulations and thermodynamic data has also been used. ${ }^{6}$ It has been shown, for pair potentials, bottom-up approaches can lead to CG models which represent local structure well, but can have large inaccuracies for thermodynamic quantities like pressure and isothermal compressibility. ${ }^{27,37}$ (This implies that the pairwise additivity assumption, which tends to work for all-atom models, is not valid at the CG level and many body terms are necessary.) The water-alkane parameters are developed using a combination method and the alkane-alkane parameters from a top-down approach.

The alkane-alkane and alkane-water interactions were taken to be described by the two-body potential only, following earlier work, ${ }^{7,48}$ since there are no hydrogen bonds or other directional interactions that are lost once the hydrogen sites are eliminated. Also following earlier work, water-solute, and solute-solute interactions are developed independently, without the use of combining rules. ${ }^{6,7,48}$ There are two carbon types, $\mathrm{CH}_{3}$ and $\mathrm{CH}_{2}$, and each atom type has two parameters associated with it, $\epsilon$ and 
$\sigma$. The cross alkane-alkane parameters, as between $\mathrm{CH}_{3}$ and $\mathrm{CH}_{2}$ sites, are given by the algebraic mean, so that $\epsilon\left(\mathrm{CH}_{3}, \mathrm{CH}_{2}\right)=(1 / 2)\left(\epsilon\left(\mathrm{CH}_{3}, \mathrm{CH}_{3}\right)+\epsilon\left(\mathrm{CH}_{2}, \mathrm{CH}_{2}\right)\right)$ and $\sigma\left(\mathrm{CH}_{3}, \mathrm{CH}_{2}\right)=(1 / 2)\left(\sigma\left(\mathrm{CH}_{3}, \mathrm{CH}_{3}\right)+\sigma\left(\mathrm{CH}_{2}, \mathrm{CH}_{2}\right)\right)$. For liquid alkanes, the parameters are chosen to best fit the density and enthalpy of vaporization, which is found from

$$
\Delta H_{v a p}=\left\langle E_{g}\right\rangle-\left\langle E_{l}\right\rangle+R T
$$

where $\left\langle\mathrm{E}_{g}\right\rangle$ is the gas-phase energy and $\left\langle\mathrm{E}_{l}\right\rangle$ is the liquid phase energy. In principle, a coarse-grained energy like $\left\langle\mathrm{E}_{l}\right\rangle$ contains an entropic part resulting from the loss of atomic detail. ${ }^{49}$ This means that potentially there is an entropic contribution which needs to be added when comparing to the experimental $\Delta H_{v a p}$. For these alkane molecules, this entropic part comes from the terminal methyl groups, since the $\mathrm{CH}_{2}$ hydrogens do not have much conformational freedom. Following a long history of previous work with united atom models, ${ }^{10,14,50,51}$ and other CG models ${ }^{23}$ we use Equation 5 without any corrections for entropy. Correctly reproducing the density and $\Delta H_{\text {vap }}$ implies the model has the correct length and energy scales, respectively, and these are common properties for alkane models to fit against. ${ }^{10,23,45,52}$ The alkanewater parameters are chosen to reproduce the solvation free energies and water-solute pair correlation functions, as compared to OPLS-AA ${ }^{53}$ and $\mathrm{SPC} / \mathrm{E}^{54}$ results.

As additional tests of the models, the decane/vapor and decane/water surface tensions, $\gamma$, are calculated, by setting up an interfacial system, using the pressure tensors,

$$
\left.\gamma=\frac{L_{z}}{2}\left[P_{z z}-\left(P_{x x}+P_{y y}\right) / 2\right)\right]
$$

where $\mathrm{z}$ is the direction perpendicular to the interface, $\mathrm{L}_{z}$ is the box length in that direction, and $\mathrm{P}_{\alpha \alpha}$ is the diagonal pressure tensor in direction $\alpha$. The shear viscosity, 
$\eta$, of liquid decane is found from the Green-Kubo relation ${ }^{55}$

$$
\eta=\frac{V}{k_{B} T} \lim _{\tau \rightarrow \infty} \int_{0}^{\tau} \frac{1}{3}\left(\left\langle P_{x y}(t) P_{x y}(0)+P_{x z}(t) P_{x z}(0)+P_{y z}(t) P_{y z}(0)\right\rangle\right) d t
$$

where $\mathrm{V}$ is volume, $k_{B}$ is Boltzmann's constant, and $\mathrm{P}_{\alpha \beta}(\mathrm{t})$ is the time-dependence of the off-diagonal pressure tensor. Values of $\eta$ are found from an average of all three off-diagonal pressure tensors. The plateau region of the integral in Equation 7 is reached around $20 \mathrm{ps}$ and this value is used to find $\eta$. The diffusion constant is found from the mean-squared displacement of the center-of-mass using the Einstein relation.

Simulation details. The CG simulations use LAMMPS, ${ }^{56}$ with a 5 fs time step in the isothermal-isobaric (TPN) ensemble, except as noted. Temperature and pressure were controlled using a Nosé-Hoover thermostat, with a 100 fs damping constant, and a barostat, with a damping parameter of $1000 \mathrm{fs}$, at a temperature of $300 \mathrm{~K}$ and a pressure of 1 bar,respectively. The liquid alkane simulations used 486 molecules for pentane and hexane and 243 molecules for alkanes larger than hexane. The aqueous alkane simulations used one alkane and 2476 water molecules. The alkane/vapor surface tension simulations used a $42.9 \AA \times 42.9 \AA \times 100 \AA$ orthorhombic box, with 286 molecules for decane and hexane, and 486 molecules for decane through hexadecane. The alkane/water surface tension calculations this same number of alkane molecules and 2476 water molecules, with the box size adjusted to give a total pressure of 1 atm. The surface tension simulations were done in the canoncal (TVN) ensemble. The calculations for the viscosity and diffusion constants were done in the microcanonical (EVN) ensemble. The free energy calculations were done using finite difference thermodynamic integration (FDTI), ${ }^{57}$ using a soft core potential to avoid singularities. ${ }^{58}$ We added the subroutine necessary to implement 
TI in LAMMPS with the SW potentials. These routines, along with sample input files, are available on github. ${ }^{59}$ The calculations used a total simulation time of $20 \mathrm{~ns}$ and used 40 equally spaced integration points along the $\lambda$ coordinate. For decane, the TI calculations used 25 ns with 50 integration points. The all-atom simulations used the Gromacs simulation package ${ }^{60}$ with the OPLS-AA model ${ }^{53}$ for the alkanes and the SPC/E water model. ${ }^{54}$

\section{Results}

The optimal alkane parameters are given in Table 1, along with previously developed parameters for water ${ }^{3}$ and methane. ${ }^{48}$ In both the alkane-alkane and alkane-water potentials, developed independently, $\sigma$ is smaller and $\epsilon$ is larger for $\mathrm{CH}_{3}$ relative to $\mathrm{CH}_{2}$, which is consistent with the results of previous all atom models. The Trappe$\mathrm{UA}^{13-15}$ and $\mathrm{HH}^{16}$ models also have $\sigma$ smaller and $\epsilon$ larger and OPLS-UA has the same $\sigma$ and a larger $\epsilon$ n-alkane for $\mathrm{CH}_{3}$ groups relative to $\mathrm{CH}_{2}$ groups.

The density and the enthalpy of vaporization for the liquid alkanes are shown in Figure 2 and Table 2, respectively. The model does very well in reproducing the experimental data, ${ }^{61}$ which are the two quantities used to find the parameters. The free energies of hydration for ethane, butane, and decane are given in Table 3. The potential can be made to fit the experimental ${ }^{62}$ free energies fairly accurately. Also shown is the result for methane, using the Song-Molinero parameters, ${ }^{48}$ and we find a value in reasonably close agreement with the reported value of $1.6 \mathrm{kcal} / \mathrm{mol}{ }^{48}$ The other properties used in the parameter optimization were the carbon-water pair correlation functions. The water-carbon radial distribution functions match those from the OPLS-UA model for the $\mathrm{CH}_{3}$ and $\mathrm{CH}_{2}$ groups (Figure 3).

An important test of the quality of the model for applications to macromolecu- 
lar assembly is the surface tension. For the alkanes both the liquid/vapor and liquid/water surface tension are accurately reproduced (Table 2 and Figure2 (C)). The alkane/vapor surface tension is in close agreement with experiment. ${ }^{63}$ The OPLS-AA model ${ }^{53}$ gives similar accuracies for alkane/vapor surface tensions, ${ }^{64-66}$ while results for united atom models vary in accuracy. ${ }^{65-70}$ The course grained model, with 4:1 mapping of heavy atoms, of Nielsen, et al., used the surface tension in the parameterization of the model, and reproduces that property well. ${ }^{19}$ The alkane/water surface tension is lower than the experimental value,${ }^{63}$ with values that are $18 \%$ lower for pentane to $7 \%$ lower for hexadecane. This is consistent with the properties of the water model. The water/vapor surface tension of the $\mathrm{mW}$ model $\left(66 \mathrm{dyn}^{-1}\right)^{3}$ is about $8 \%$ lower than experiment $\left(71.6 \mathrm{dyn} \mathrm{cm}^{-1}\right) .{ }^{71}$ Most common water models underestimate the surface tension by about the same amount as the mW model, ${ }^{72}$ except for some polarizable models. ${ }^{73}$ Qui and Molinero ${ }^{70}$ using OPLS-UA and $\mathrm{mW}$ water found a nonane/water surface tension in good agreement with experiment. ${ }^{69}$ All atoms models give water/alkane surface tensions about $16 \%$ off, both lower ${ }^{74}$ and higher ${ }^{75}$ than the experimental value.

Table 4 shows the gauche-trans fraction for the middle and end dihedrals. Infrared spectroscopy experiments find that, for tridecane, the fraction of gauche is about 0.33 for the middle and 0.44 for the end torsions. ${ }^{46}$ Our model is in good agreement with these results, indicating that fitting to the hexane torsional energy profile of Siu et al. ${ }^{45}$ gives the correct amount of backbone flexibility, as it did for all-atom models. ${ }^{45}$ Models that over-stabilize trans conformations tend to form gel-like states for longer alkanes, so getting the gauche/trans ratio correct is important. ${ }^{45}$ The radius of gyration, $\mathrm{R}_{G}$, is another measure of the conformation of the alkanes (Table 4 and Figure $2(\mathrm{D})$ ). The $\mathrm{R}_{G}$ is almost the same in the liquid alkane and aqueous phases, with only a small decrease in water, indicating that there is no transition 
to a compact structure. A fit to the results indicates that $\mathrm{R}_{G}$ increases with the number of carbons, $N_{C}$, as $N_{C}^{0.85}$, so slightly less than the increase for a rigid linear chain. Simulations with all atom ${ }^{19,76,77}$ and $\mathrm{CG}^{19}$ models show a similar $\mathrm{R}_{G}$, with a similar size dependence. Ferguson, et al., also find essentially no dependence of $\mathrm{R}_{G}$ on phase. ${ }^{77}$

Dynamical properties of the model are determined using the shear viscosity for the liquid alkanes and diffusion constants for the small alkanes in water. The shear viscosity, found from Equation 7, is almost a factor of two less than the experimental value $^{78}$ for decane (Table 1) and about a factor of 3 less for tetradecane, indicating faster dynamics of the model. The aqueous diffusion constants are about a factor of two more than the experimental values ${ }^{79}$ (Table 5). Similar SW models for methane in $\mathrm{mW}$ water find a methane diffusion constant of $4.8 \times 10^{5} \mathrm{~cm}^{2} / \mathrm{sec},{ }^{7}$ larger than the experimental value $\left(1.88 \times 10^{5} \mathrm{~cm}^{2} / \mathrm{sec}^{79}\right)$ and the $\mathrm{mW}$ water model overes-

timates the diffusion constant by just over a factor of two $\left(6.5 \times 10^{5} \mathrm{~cm}^{2} / \mathrm{sec}\right.$ versus $\left.2.3 \times 10^{5} \mathrm{~cm}^{2} / \mathrm{sec}^{3}\right)$. Course-grained models in general tend to move on faster times scales, ${ }^{24}$ as the removal of atomistic detail smoothens the potentials, reducing the friction between particles. For processes in water, dynamics is influenced by hydrogen bond re-orientations, which are not present in the $\mathrm{mW}$, hydrogen-less, model, leading to faster dynamics. ${ }^{3}$ If the goal of the CG model is thermodynamics and structure, and not dynamics, the speed ups can be taken as a benefit, as equilibrium will be reached sooner.

\section{Conclusions}

The SW potential can reproduce many important properties of liquid alkanes and aqueous alkanes. The strength of the alkane-alkane interactions is verified by an 
accurate enthalpy of vaporization and decane/vapor surface tension. The liquid decane/water surface tension and the hydration free energies indicate that the alkanewater interactions have the correct magnitude. The length scales for the interactions are validated by the liquid alkane densities and the positions of the water-carbon radial distribution functions. The time scales from the liquid alkane viscosity and aqueous diffusion constants are about a factor two faster than experiment, consistent with previous results using the SW models. ${ }^{3,7}$

The alkane models have larger $\sigma$ parameters than water and so are longer-ranged. The two-body potential goes to zero at a distance of a times $\sigma$ (Eq. 2), which equals about 8 to $9 \AA$ for the alkane-alkane interactions. This is a distance just beyond the nearest neighbors (Figure 4). (In terms of structure, the alkane interactions are shorter-ranged than the water-water interactions, which go to zero at $4.31 \AA$, corresponding to a distance well into the second solvation shell.) The larger $\sigma$ as well as the more close packed structure of the alkanes does make the gains in efficiency less than those of liquid water. For liquid decane, each decane atom interacts with about 38 atoms, including atoms on the same decane molecule. With a $12 \AA$ cut-off, each atom would interact with 95 heavy atoms, so the reduced range model results in 2.5 fewer interactions. For decane-water interactions, each decane atom interactions with 64 water molecules, while 239 are within $12 \AA]$, so there are 3.7 fewer interactions. For the alkane SW model, then, there are about a factor of 3 fewer interactions due to the reduced range of the potential. An all-atom alkane model would have about twice as many atoms, so there is an additional factor of four decrease in the computational cost from coarse-graining the hydrogen sites.

The SW model presents a relatively accurate and efficient method for treating long alkanes, in agreement with previously developed models for water, ${ }^{3}$ methane, ${ }^{7}$ and aqueous sodium chloride. ${ }^{6}$ The approach represents an intermediate level of coarse- 
graining between all-atom and larger 4:1 type models. The SW model gains speed from the lack of long-ranged interactions, so it does not require Ewald sums or the equivalent and has fewer interacting neighbors. The use of shorter-ranged potentials, rather than larger scale coarse-graining, to create faster models presents some advantages. It retains a molecular level description and does not require combining atoms into sometimes arbitrary groups. In addition, for multiscale simulations which convert between all-atom (AA) and CG models, the backward mapping problem (CG to AA) becomes simpler.

\section{Acknowledgements}

This work is supported by the U.S. Department of Energy under EPSCoR Grant No. DE-SC0012432 with additional support from the Louisiana Board of Regents. We acknowledge the Louisiana Optical Network Initiative (LONI) for computer time and thank Valeria Molinero for useful discussions.

\section{Supporting information}

LAMMPS input files for a decane/water mixture are provided as supplementary material. This information is available free of charge via the Internet at http://pubs.acs.org

\section{References}

(1) Krieg, E.; Bastings, M. M.; Besenius, P.; Rybtchinski, B. Chem. Rev. 2016, $116,2414-2477$.

(2) Stillinger, F. H.; Weber, T. A. Physical Review B 1986, 31, 5262-5271. 
(3) Molinero, V.; Moore, E. B. J. Phys. Chem. B 2009, 113, 4008-4016.

(4) Larini, L.; Lu, L.; Voth, G. A. J. Chem. Phys. 2010, 132, 164107.

(5) Das, A.; Andersen, H. C. J. Chem. Phys. 2012, 136, 194114.

(6) DeMille, R. C.; Molinero, V. J. Chem. Phys. 2009, 131, 034107.

(7) Jacobson, L. C.; Molinero, V. J. Phys. Chem. B 2010, 114, 7302-7311.

(8) Ryckaert, J. P.; Bellemans, A. Faraday Discuss. Chem. Soc. 1978, 66, 95.

(9) Weber, T. A. J. Chem. Phys. 1978, 69, 2347.

(10) Jorgensen, W. L.; Madura, J. D.; Swenson, C. J. J. Am. Chem. Soc. 1984, 106, 6638-6646.

(11) Siepmann, J. I.; Karaborni, S.; Smit, M. J. Chem. Phys. 1993, 365, 330.

(12) Dodd, L. R.; Theodorou, D. N. Adv. Polymer Sci. 1994, 116, 249.

(13) Martin, M. G.; Siepmann, J. I. J. Phys. Chem. B 1998, 102, 2569-2577.

(14) Wick, C. D.; Martin, M. G.; Siepmann, J. I. J. Phys. Chem. B 2000, 104, 8008-8016.

(15) Martin, M. G.; Siepmann, J. I. J. Phys. Chem. B 1999, 103, 4058-4517.

(16) Ashbaugh, H. S.; Liu, L.; Surampdi, L. N. J. Chem. Phys. 2011, 135, 054510.

(17) Yang, L.; Tan, C. H.; Hseih, M. J.; Wang, J.; Cieplak, P.; Caldwell, J.; Kollman, P. A.; Luo, R. J. Phys. Chem. B 2006, 110, 13166-13176.

(18) Shelley, J. C.; Shelley, M. Y.; Reeder, R. C.; Bandyopadhyay, S.; Klein, M. L. J. Phys. Chem. B 2001, 105, 4464-4470. 
(19) Nielsen, S. O.; Lopez, C. F.; Srinivas, G.; Klein, M. L. J. Chem. Phys. 2003, 119, 7043-7049.

(20) Marrink, S. J.; de Vries, A. H.; Mark, A. E. J. Phys. Chem. B 2004, 108, $750-760$.

(21) Izvekov, S.; Voth, G. A. J. Phys. Chem. B 2005, 109, 2469-2473.

(22) Markvoort, A. J.; Pieterse, K.; Steijaert, M. N.; Hilbers, P. A. J. J. Phys. Chem. B 2005, 109, 22649-22654.

(23) Chiu, S. W.; Scott, H. L.; Jakobsson, E. J. Chem. Theory Comput. 2010, 6, $851-863$.

(24) Marrink, S. J.; Tieleman, D. P. Chem. Soc. Rev. 2013, 42, 6801.

(25) Izvekov, S.; Swanson, J. M. J.; Voth, G. A. J. Phys. Chem. B 2008, 112, 47114724 .

(26) Guenza, M. Eur. Phys. J.: Spec. Top. 2015, 224, 2177-2191.

(27) Dunn, N. J. H.; Noid, W. G. J. Chem. Phys. 2015, 143, 243148.

(28) Andersen, H. C.; Chandler, D.; Weeks, J. D. Adv. Chem. Phys. 1976, 34, 105.

(29) Adams, D. J. Chem. Phys. Lett. 1979, 62, 329-332.

(30) Linse, P.; Andersen, H. C. J. Chem. Phys. 1986, 85, 3027-3041.

(31) Perera, L.; Essmann, U.; Berkowitz, M. L. J. Chem. Phys. 1995, 102, 450-456.

(32) Ashbaugh, H.; Wood, R. J. Chem. Phys. 1997, 106, 8135-8139. 
(33) Feller, S. E.; Pastor, R. W.; Rojnuckarin, A.; Bogusz, S.; Brooks, B. R. J. Phys. Chem. 1996, 100, 17011-17020.

(34) Spohr, E. J. Chem. Phys. 1998, 107, 6342.

(35) Rogers, J. M.; Weeks, J. D. J. Phys.: Condens. Matter 2008, 20, 494206.

(36) Rogers, J. M.; Weeks, J. D. Proc. Natl. Acad. Sci. 2008, 105, 19136-19141.

(37) Johnson, M. E.; Head-Gordon, T.; Louis, A. A. J. Chem. Phys. 2007, 126, 144509.

(38) Wang, H.; Junghans, C.; Kremer, K. Eur. Phys. J 2009, 28, 221-229.

(39) Tan, M. L.; Fischer, J. T.; Chandra, A.; Brooks, B. R.; Ichiye, T. Chem. Phys. Lett. 2003, 376, 646-652.

(40) Das, A.; Andersen, H. C. J. Chem. Phys. 2010, 132, 164106.

(41) Allen, E. C.; Rutledge, G. C. J. Chem. Phys. 2008, 128, 154115.

(42) Ilie, I. M.; den Otter, W. K.; Briels, W. J. J. Chem. Phys. 2016, 144, 085103.

(43) Dama, J. E.; Jin, J.; Voth, G. A. J. Chem. Theory Comput. 2017, 13, 10101022.

(44) Jacobson, L. C.; Kirby, R. M.; Molinero, V. J. Phys. Chem. B 2014, 118, 8190-8202.

(45) Siu, S. W.; Pluhackova, K.; Böckmann, R. A. J. Chem. Theory Comp. 2012, 8, $1459-1470$.

(46) Casal, H. L.; Mantsch, H. H. J. Mol. Struct. 1989, 192, 41-45. 
(47) Lu, J.; Qiu, Y.; Baron, R.; Molinero, V. J. Chem. Theory Comput. 2014, 10, 4104-4120.

(48) Song, B.; Molinero, V. J. Chem. Phys. 2013, 139, 054511.

(49) Foley, T. T.; Shell, M. S.; Noid, W. G. J. Chem. Phys. 2015, 143, 243104.

(50) Jorgensen, W. L.; Briggs, J. M.; Contreras, M. L. J. Phys. Chem. 1990, 94, $1683-1686$.

(51) Qiu, Y.; Molinero, V. Crystals 2017, 7, 86.

(52) Dickson, C. J.; Madej, B. D.; Skevik, A. A.; Betz, R. M.; Teigen, K.; Gould, I. R.; Walker, R. C. J. Chem. Theory Comput. 2014, 10, 865-879.

(53) Jorgensen, W. L.; Maxwell, D. S.; Tirado-Rives, J. J. Am. Chem. Soc. 1996, $118,11225-11236$.

(54) Berendsen, H. J. C.; Grigera, J. R.; Straatsma, T. P. J. Phys. Chem. 1987, 91, 6269.

(55) Cui, S. T.; Cummings, P. T.; Cochran, H. D. Molec. Phys. 1998, 93, 117-121.

(56) Plimpton, S. Journal of Computational Physics 1995, 117, 1-19.

(57) Mezei, M. J. Chem. Phys. 1987, 86, 7084-7088.

(58) Beutler, T. C.; Mark, A. E.; van Schaik, R. C.; Gerber, P. R.; van Gunsteren, W. F. Chem. Phys. Lett. 1994, 222, 529-539.

(59) A git repository for the free energy routines is hosted at https://github.com/ ggyawali/pair_sw_soft/tree/master. 
(60) van der Spoel, D.; Lindahl, E.; Hess, B.; Groenhof, G.; Mark, A. E.; Berendsen, H. J. C. J. Comp. Chem. 2005, 26, 1701-1718.

(61) Weast, R. C., Ed. CRC Handbook of Chemistry and Physics; CRC Press: Boca Raton, Florida, 2013; Vol. 94th Edition.

(62) Abraham, M. H.; Whiting, G. S.; Fuchs, R.; Chambers, E. J. J. Chem. Soc.Perkin Trans. 1990, 2, 291-300.

(63) Goebel, A.; Lunkenheimer, K. Langmiur 1997, 13, 369-372.

(64) Pierce, F.; Tsige, M.; Borodin, O.; Perahia, D.; Grest, G. S. J. Chem. Phys. 2008, 128, 214903.

(65) Amat, M. A.; Rutledge, G. C. J. Chem. Phys. 2010, 132, 114704.

(66) Bresme, F.; Chacócon, E.; Tarazona, P. Molecular Physics 2010, 108, 18871898.

(67) Hariharan, A.; Harris, J. G. J. Chem. Phys. 1994, 101, 4156.

(68) Biscay, F.; Ghoufi, A.; Goujon, F.; Lachet, V.; Malfreyt, P. J. Phys. Chem. B 2008, 112, 13885-13897.

(69) Mendoza, F. N.; López-Rendón, R.; López-Lemus, J.; Cruz, J.; Alejandre, J. Molecular Physics 2008, 106, 1055-1059.

(70) Qiu, Y.; Molinero, V. J. Am. Chem. Soc. 2015, 137, 10642-10651.

(71) Vargaftik, N. B.; Volkov, B. N.; Voljak, L. D. J. Phys. Chem. Ref. Data 1983, 12, 817 .

(72) Vega, C.; de Miguel, E. J. Chem. Phys. 2007, 126, 154707. 
(73) Rick, S. W. J. Comput. Chem. 2016, 37, 2060-2066.

(74) Patel, S. A.; Brooks, III, C. L. J. Chem. Phys. 2006, 124, 204706.

(75) Zhang, Y.; Feller, S. E.; Brooks, B. R. J. Chem. Phys. 1995, $103,10252$.

(76) Mountain, R. D.; Thirumalai, D. Proc. Natl. Acad. Sci. (USA) 1998, 95, 84368440.

(77) Ferguson, A. L.; Debenedetti, P. G.; Panagiotopoulos, A. Z. J. Phys. Chem. B 2009, 113, 6405-6414.

(78) Dymond, J. H.; Øye, H. A. J. Phys. Chem. Ref. Data 1994, 23, 41-53.

(79) Witherspoon, P. A.; Saraf, D. N. J. Phys. Chem. 1965, 69, 3752-3755. 
Table 1: Two-body potential parameters for alkanes and previously published parameters for methane ${ }^{48}$ and water. ${ }^{3}$

\begin{tabular}{lll}
\hline \hline & $\sigma(\AA)$ & $\epsilon(\mathrm{kcal} / \mathrm{mol})$ \\
\hline $\mathrm{CH}_{4} \mathrm{CH}_{4}$ & 4.08 & 0.390 \\
$\mathrm{CH}_{3} \mathrm{CH}_{3}$ & 4.64 & 0.100 \\
$\mathrm{CH}_{2} \mathrm{CH}_{2}$ & 5.08 & 0.072 \\
$\mathrm{CH}_{4} \mathrm{H}_{2} \mathrm{O}$ & 4.05 & 0.239 \\
$\mathrm{CH}_{3} \mathrm{H}_{2} \mathrm{O}$ & 4.25 & 0.165 \\
$\mathrm{CH}_{2} \mathrm{H}_{2} \mathrm{O}$ & 4.29 & 0.1222 \\
$\mathrm{H}_{2} \mathrm{O} \mathrm{H}_{2} \mathrm{O}$ & 2.3925 & 6.189 \\
\hline \hline
\end{tabular}

Table 2: Density, enthalpy of vaporization, surface tensions, and viscosities for liquid alkanes. Experimental data for the density and enthalpy of vaporization is from Reference 61, surface tension from Reference 63, and viscosities from Reference 78.

\begin{tabular}{|c|c|c|c|c|c|}
\hline & \multirow{2}{*}{$\begin{array}{l}\text { density } \\
\left(\mathrm{kg} / \mathrm{m}^{3}\right)\end{array}$} & \multirow{2}{*}{$\begin{array}{l}\Delta \mathrm{H}_{\text {vap }} \\
(\mathrm{kcal} / \mathrm{mol})\end{array}$} & \multicolumn{2}{|c|}{ surface tension $\left(\right.$ dyn $\left.\mathrm{cm}^{-1}\right)$} & \multirow{2}{*}{$\begin{array}{l}\text { viscosity } \\
(\mathrm{mPa} \mathrm{s})\end{array}$} \\
\hline & & & liquid/vapor & liquid/water & \\
\hline pentane & $618.6 \pm 0.9$ & $6.5 \pm 0.1$ & $14 \pm 2$ & $42 \pm 1$ & $0.18 \pm 0.01$ \\
\hline experiment & 626.6 & 6.32 & 15.9 & 50.9 & \\
\hline hexane & $656.7 \pm 0.8$ & $7.7 \pm 0.1$ & $18 \pm 2$ & $43 \pm 1$ & $0.22 \pm 0.01$ \\
\hline experiment & 660.6 & 7.57 & 18.3 & 51.4 & 0.2949 \\
\hline octane & $702.8 \pm 0.7$ & $10.1 \pm 0.1$ & $22 \pm 2$ & $46 \pm 1$ & $0.35 \pm 0.02$ \\
\hline experiment & 698.6 & 9.92 & 21.55 & 52.5 & 0.5092 \\
\hline decane & $728.9 \pm 0.6$ & $12.5 \pm 0.1$ & $24 \pm 2$ & $46 \pm 1$ & $0.46 \pm 0.02$ \\
\hline experiment & 726.7 & 12.3 & 23.7 & 53.2 & 0.8498 \\
\hline dodecane & $747.9 \pm 0.9$ & $14.7 \pm 0.1$ & $25 \pm 2$ & $47 \pm 1$ & $0.57 \pm 0.03$ \\
\hline experiment & 749.5 & 14.7 & 25.3 & 53.7 & 1.3585 \\
\hline tetradecane & $759.5 \pm 0.9$ & $17.1 \pm 0.1$ & $26 \pm 2$ & $49 \pm 1$ & $0.66 \pm 0.04$ \\
\hline experiment & 759.6 & 17.1 & 26.4 & 54.5 & 2.078 \\
\hline hexadecane & $768.9 \pm 0.7$ & $19.5 \pm 0.1$ & $28 \pm 2$ & $50 \pm 1$ & $0.78 \pm 0.04$ \\
\hline experiment & 770.1 & 19.4 & 27.2 & 55.2 & \\
\hline
\end{tabular}


Table 3: Hydration free energies for selected alkanes. The experimental data is from Reference 62.

\begin{tabular}{lll}
\hline \hline & \multicolumn{2}{c}{$\Delta \mathrm{G}_{\text {solv }}(\mathrm{kcal} / \mathrm{mol})$} \\
& model & experiment \\
\hline methane & $2.11 \pm 0.03$ & 1.99 \\
ethane & $1.88 \pm 0.05$ & 1.83 \\
butane & $2.02 \pm 0.06$ & 2.07 \\
hexane & $2.7 \pm 0.2$ & 2.47 \\
octane & $3.0 \pm 0.4$ & 2.88 \\
decane & $3.0 \pm 0.7$ & 3.16 \\
\hline \hline
\end{tabular}

Table 4: Conformation properties of the alkanes, gauche-trans fractions of the dihedral angles for the pure liquid and the radius of gyration for the liquid and aqueous phases.

\begin{tabular}{lllll}
\hline \hline & \multicolumn{2}{c}{ gauche-trans fraction } & \multicolumn{2}{c}{$\mathrm{R}_{G}(\AA)$} \\
& end & middle & pure liquid & aqueous \\
\hline pentane & & $0.430 \pm 0.004$ & $1.75 \pm 0.01$ & $1.72 \pm 0.01$ \\
hexane & $0.407 \pm 0.003$ & $0.432 \pm 0.005$ & $2.06 \pm 0.01$ & $2.03 \pm 0.01$ \\
octane & $0.390 \pm 0.004$ & $0.441 \pm 0.008$ & $2.64 \pm 0.01$ & $2.64 \pm 0.01$ \\
decane & $0.385 \pm 0.003$ & $0.447 \pm 0.008$ & $3.20 \pm 0.01$ & $3.18 \pm 0.01$ \\
dodecane & $0.385 \pm 0.003$ & $0.450 \pm 0.007$ & $3.72 \pm 0.01$ & $3.69 \pm 0.01$ \\
tetradecane & $0.382 \pm 0.003$ & $0.451 \pm 0.009$ & $4.22 \pm 0.01$ & $4.19 \pm 0.02$ \\
hexadecane & $0.381 \pm 0.001$ & $0.450 \pm 0.005$ & $4.68 \pm 0.01$ & $4.64 \pm 0.02$ \\
\hline \hline
\end{tabular}

Table 5: Aqueous diffusion constants for selected alkanes. The experimental data is from Reference 79.

\begin{tabular}{lll}
\hline \hline & \multicolumn{2}{c}{$\mathrm{D}\left(\times 10^{5} \mathrm{~cm}^{2} / \mathrm{sec}\right)$} \\
& model & experiment \\
\hline ethane & $2.9 \pm 0.2$ & $1.52 \pm 0.03$ \\
propane & $2.8 \pm 0.2$ & $1.21 \pm 0.04$ \\
butane & $2.6 \pm 0.2$ & $0.96 \pm 0.04$ \\
\hline \hline
\end{tabular}




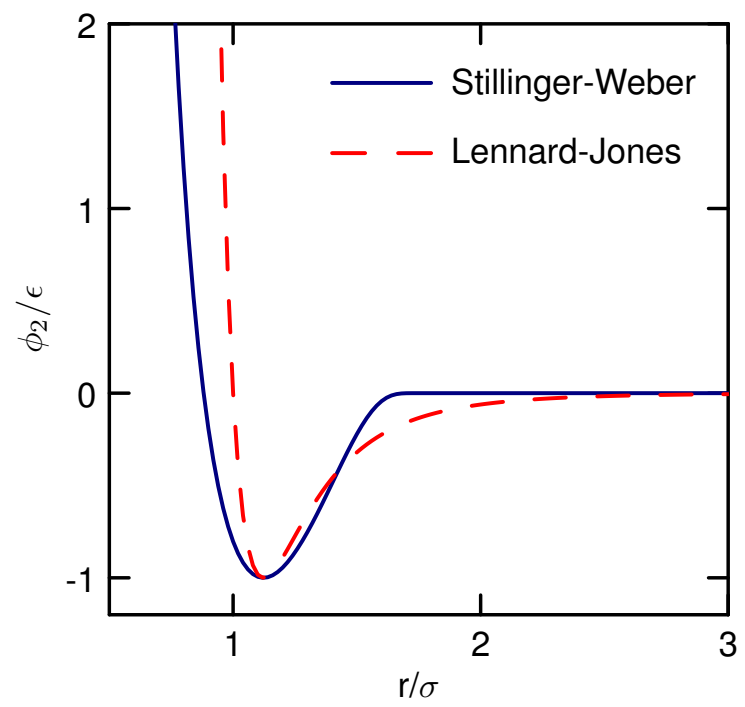

Figure 1: The two-body Stillinger-Weber and Lennard-Jones potentials. 

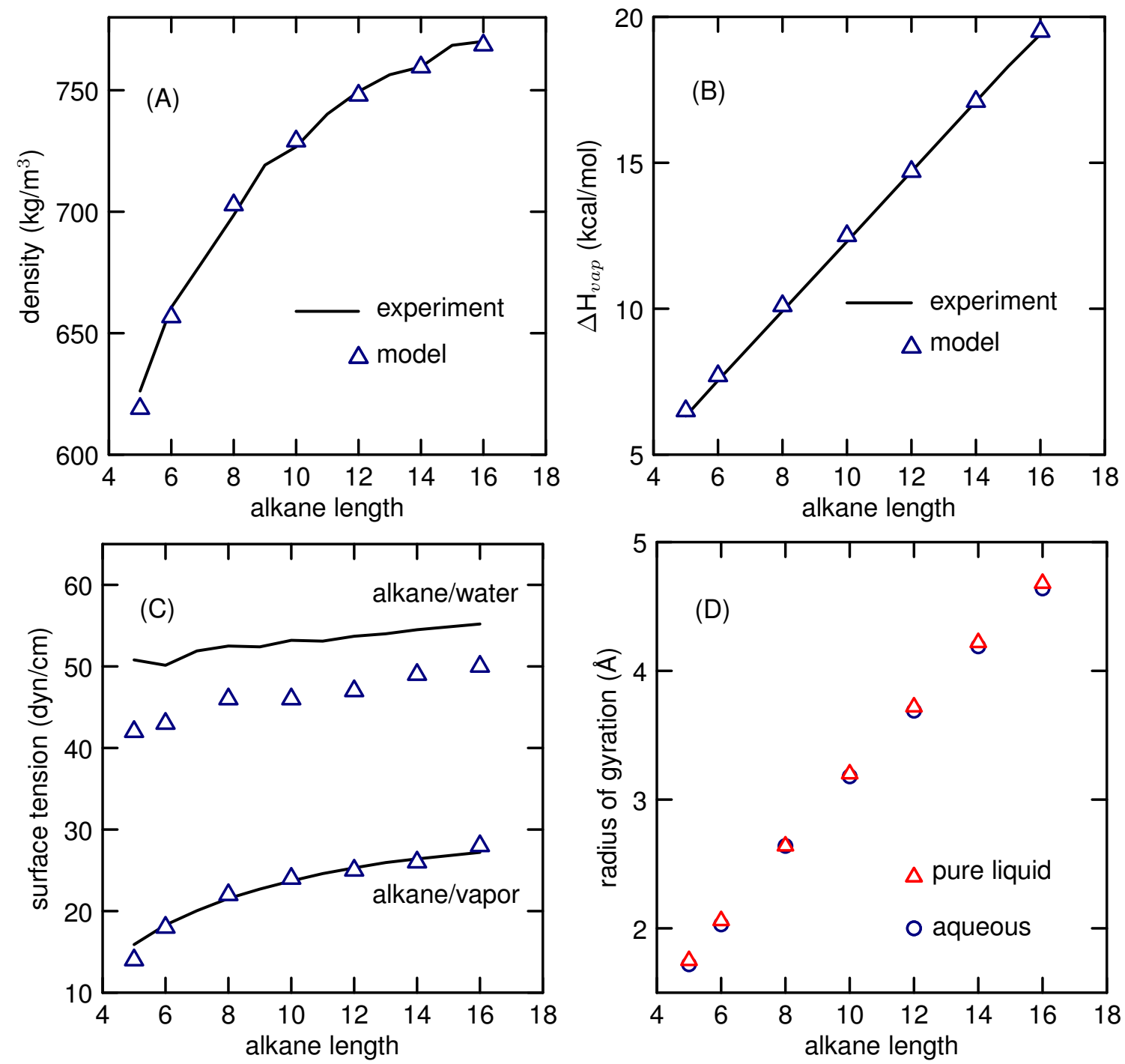

Figure 2: The density (A),enthalpy of vaporization (B), surface tensions (C), and radius of gyration (D) of liquid alkanes, comparing the model (symbols) and experiment (solid lines). Experimental data for the density and enthalpy of vaporization is from Reference 61, and the surface tension data is from Reference 63. 

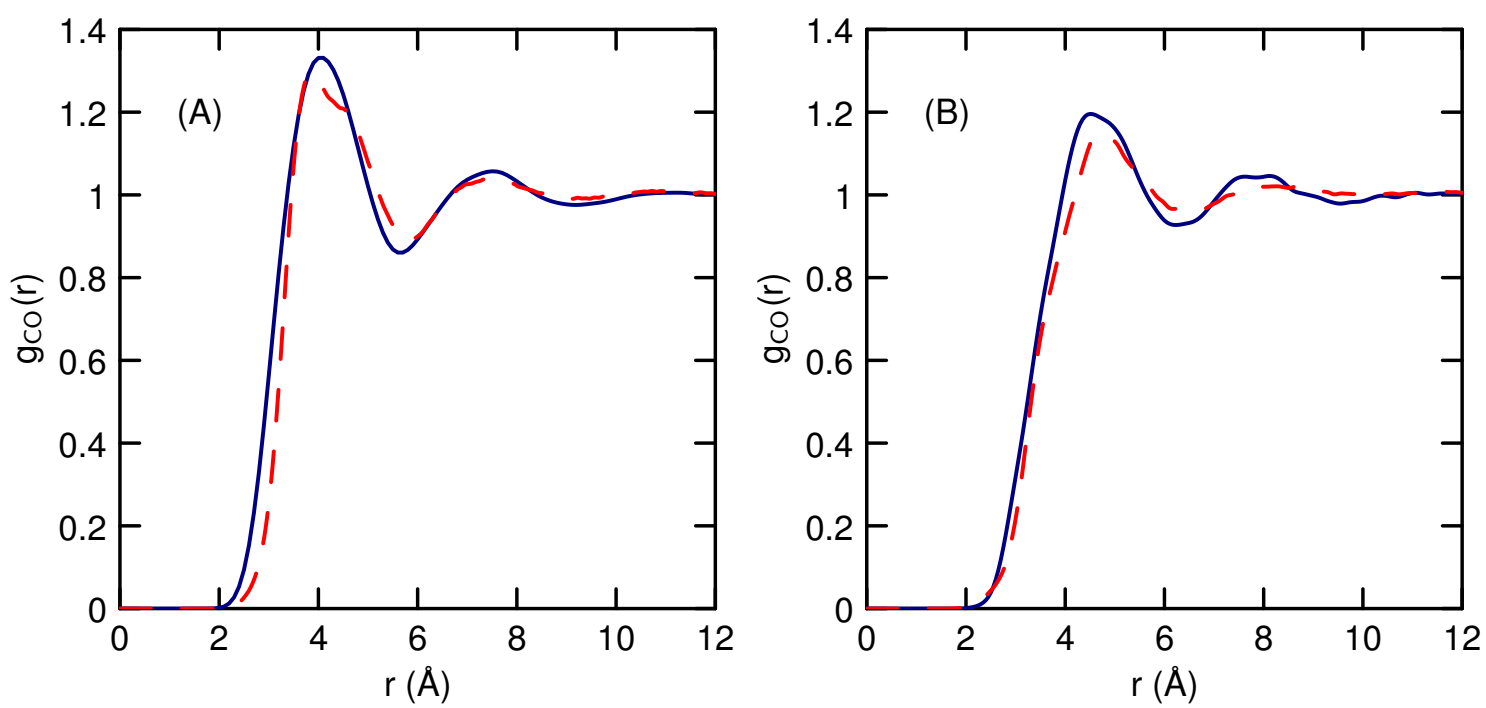

Figure 3: Water oxygen-ethane $\mathrm{CH}_{3}(\mathrm{~A})$ and water oxygen-butane $\mathrm{CH}_{2}$ (B) radial distribution functions comparing the CG (solid line) and OPLS-UA models (dashed line). 


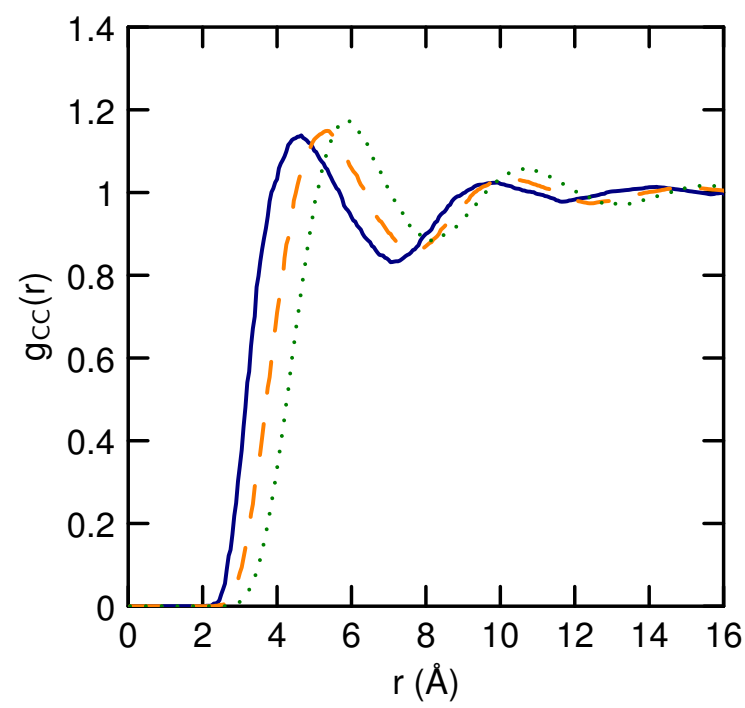

Figure 4: Liquid decane inter-molecular radial distribution functions for $\mathrm{CH}_{3}-\mathrm{CH}_{3}$ atoms (solid line), $\mathrm{CH}_{3}-\mathrm{CH}_{2}$ atoms (dashed line), and $\mathrm{CH}_{2}-\mathrm{CH}_{2}$ atoms (dotted line) 